\title{
PRODUÇÃO DE POLI(3-HIDROXIBUTIRATO) POR Cupriavidus necator EM MEIO HIDROLISADO DE AMIDO DE ARROZ COM SUPLEMENTAÇÃO DE ÓLEO DE SOJA EM DIFERENTES TEMPERATURAS
}

\author{
Francieli Dalcanton, Jaciane Lutz Ienczak, Mônica Lady Fiorese e Gláucia Maria Falcão de Aragão* \\ Departamento de Engenharia Química e Engenharia de Alimentos, Universidade Federal de Santa Catarina, CP 476, 88040-900 \\ Florianópolis - SC, Brasil
}

Recebido em 19/2/09; aceito em 7/10/09; publicado na web em 24/2/10

\begin{abstract}
PRODUCTION OF POLY(3-HYDROXYBUTYRATE) BY Cupriavidus necator IN HYDROLYZED RICE STARCH MEDIUM WITH SOYBEAN OIL SUPPLEMENTATION AT DIFFERENT TEMPERATURES. Poly (3-hydroxybutyrate) (P(3HB)) is a biopolymer, completely biodegradable, which has similar properties to fuel-based polymers. However to make it economically competitive it is necessary the study of cheap sources of substrate. The influence of hydrolyzed rice starch supplemented with soybean oil at different temperatures $\left(30,35\right.$ and $\left.40^{\circ} \mathrm{C}\right)$ was studied in the production of $\mathrm{P}(3 \mathrm{HB})$ by $C$. necator. The percentage of $\mathrm{P}(3 \mathrm{HB})$ produced in the cultures at $30,35^{\circ} \mathrm{C}$ was $30,39 \%$ and $35,43 \%$ without and with supplementation of oil, respectively. The culture at $40{ }^{\circ} \mathrm{C}$ showed no production phase due to a possible oxygen limitation. These results demonstrate that hydrolyzed rice starch supplemented with soybean oil increases the yield of $\mathrm{P}(3 \mathrm{HB})$ and temperature of $35^{\circ} \mathrm{C}$ is the most favorable for biopolymer production.
\end{abstract}

Keywords: poly(3-hydroxybutyrate); soybean oil supplementation; hydrolyzed rice starch.

\section{INTRODUÇÃO}

Em resposta à crescente preocupação com os problemas ambientais causados pela produção e acúmulo de materiais plásticos de origem petroquímica, diversos países buscam alternativas que minimizem esse impacto ambiental. Dentre as alternativas estudadas, destacam-se os poliésteres biológicos e biodegradáveis como os poli-hidroxialcanoatos (PHAs). Estes biopolímeros podem ser produzidos a partir de matérias-primas renováveis, oferecendo a possibilidade de um ciclo de produção fechado, ou seja, a fonte de carbono metabolizada pelo microorganismo produz um biopolímero completamente biodegradável a gás carbônico e água. Por conseguinte, estes elementos serão absorvidos por plantas que, novamente, fornecerão a fonte de carbono necessária para a produção dos poli-hidroxialcanoatos, fechando o ciclo. O poli(3hidroxibutirato) (P(3HB)) é o biopolímero mais estudado entre os poli-hidroxialacanoatos devido às características de biodegradabilidade e propriedades termoplásticas e mecânicas próximas às do polipropileno. ${ }^{1-3}$ Muitos são os micro-organismos produtores de $\mathrm{P}(3 \mathrm{HB})$ e a espécie Cupriavidus necator é uma das que apresenta condições mais favoráveis à produção industrial. Este micro-organismo pode acumular cerca de $80 \%$ de sua massa seca em polímero e utilizar diferentes tipos de substratos, como glicose, frutose, entre outros. ${ }^{3}$

Apesar da grande possibilidade de aplicações dos poli-hidroxialcanoatos como substituinte aos plásticos convencionais (áreas médica, farmacêutica e alimentícia e na indústria química), ${ }^{4}$ os plásticos biodegradáveis ainda têm participação mínima no mercado, em virtude de seu alto custo em relação aos plásticos petroquímicos. Desta forma, estudos que possam diminuir o custo total destes biopolímeros e também aumentar a sua produtividade são necessários.

Uma alternativa interessante para economia global do processo é a utilização de resíduos orgânicos de baixo custo, provenientes de plantas de processamento de alimentos. A utilização de substratos de baixo custo pode representar $40 \%$ de economia no processo de produção de PHAs. ${ }^{5}$ Uma fonte de carbono disponível em grandes quantidades no Brasil é o amido. ${ }^{6}$ Amido e seus derivados são os principais componentes da demanda bioquímica de oxigênio de

\footnotetext{
*e-mail: glaucia@enq.ufsc.br
}

efluentes de indústrias têxteis, fermentativas e de processamento de alimentos e bebidas. ${ }^{7}$ Nas indústrias de alimentos, o amido pode ser obtido de grãos como arroz, trigo e milho, e tubérculos como batata e mandioca. ${ }^{8}$ Determinados micro-organismos, como Cupriavidus necator, não possuem capacidade de utilizar o amido como fonte de carbono. Por isso, antes do processo biotecnológico, o amido deve ser hidrolisado a glicose. ${ }^{5,9}$ Rusendi e Sheppard ${ }^{9}$ utilizaram resíduo da indústria processadora de batatas para a produção de $\mathrm{P}(3 \mathrm{HB})$ e, através de uma hidrólise enzimática, obtiveram glicose como fonte de carbono. Kim e Chang ${ }^{10}$ estudaram o uso de amido hidrolisado como fonte de carbono para as culturas de Azotobacter chroococcum, obtendo, em batelada alimentada, uma concentração de células totais de $54 \mathrm{~g} \mathrm{~L}^{-1}$ com $46 \%$ de $\mathrm{P}(3 \mathrm{HB})$ intracelular, sob limitação em oxigênio.

No que se refere ao aumento da produtividade, a utilização de suplementos nutricionais ou indutores pode interferir na via metabólica do micro-organismo, proporcionando maior produção de células e agindo diretamente na síntese do polímero. ${ }^{11-13}$ Estudos de suplementação de cultivos utilizando os ácidos oleico e linoleico demonstraram que a adição destes ácidos graxos leva a um aumento na produção de PHAs. ${ }^{13}$ Estes ácidos graxos fazem parte da composição de muitos óleos vegetais tornando-os uma possível alternativa de baixo custo para suplementação de cultivos. Squio e Aragão ${ }^{13}$ estudaram a utilização de óleos vegetais como suplementos nutricionais. Os resultados obtidos mostraram que o uso destes óleos na produção de $\mathrm{P}(3 \mathrm{HB})$ por C. necator levou a um aumento da produção de polímero. De acordo com estes autores, as melhores alternativas de substituição dos ácidos oleico e linoleico foram os óleos de canola e oliva.

A temperatura referenciada na literatura como mais adequada para o cultivo de $C$. necator na produção de $\mathrm{P}(3 \mathrm{HB})$ é de $30{ }^{\circ} \mathrm{C} \cdot{ }^{14-16}$ No entanto, sabe-se que as enzimas presentes neste micro-organismo, assim como em outros micro-organismos, apresentam atividade máxima quando fatores, como a temperatura, se aproximam do seu ótimo, podendo haver o aumento da velocidade específica de crescimento e, consequentemente, mudanças favoráveis no fator de conversão de substrato em produto. ${ }^{17}$ Esse aumento da temperatura do cultivo pode ainda ter influência significativa na produtividade do processo, devido à diminuição do tempo de cultivo. Porém, de acordo com Schimdell, ${ }^{18}$ temperaturas de cultivo muito elevadas diminuem a concentração de 
oxigênio dissolvido no meio, fator este importante na produção de $\mathrm{P}(3 \mathrm{HB})$ por $C$. necator, já que se trata de um processo aeróbio. Neste contexto, o estudo da influência da temperatura de cultivo sobre a produção de $\mathrm{P}(3 \mathrm{HB})$, mostra-se de extrema importância, visto que na literatura há poucos relatos de trabalhos utilizando temperaturas superiores a $30{ }^{\circ} \mathrm{C}$ para a produção de $\mathrm{P}(3 \mathrm{HB})$ por $C$. necator. $\mathrm{O}$ aumento da temperatura implicaria ainda em uma menor necessidade de resfriamento durante o processo.

Este trabalho teve como objetivo estudar a influência da suplementação de óleo de soja na produção de poli(3-hidroxibutirato) por $C$. necator usando amido de arroz hidrolisado como fonte de carbono, simulando um efluente da indústria amilácea, em diferentes temperaturas de cultivo.

\section{PARTE EXPERIMENTAL}

\section{Material experimental}

Os reagentes utilizados na preparação do meio mineral foram todos da marca Vetec. As soluções de ácido e base foram preparadas por meio de reagentes da marca Nuclear. O caldo nutriente empregado na reativação do micro-organismo foi da marca Difco. Poli(3-hidroxibutirato) e ácido dinitrossalicílico foram da marca Sigma. A cepa de Cupriavidus necator DSM 545 foi adquirida na Coleção Alemã de Micro-organismo e Culturas Celulares (Deutsche Sammlung von Mikroorganismen und Zelkulturen - Braunschweig - Alemanha).

\section{Micro-organismo e meio de cultura}

A cepa utilizada foi Cupriavidus necator DSM 545.

Os experimentos foram realizados utilizando dois pré-cultivos. No primeiro, as células foram inoculadas em caldo nutriente $(\mathrm{CN})$, contendo peptona de carne $\left(5,0 \mathrm{~g} \mathrm{~L}^{-1}\right)$ e extrato de carne $\left(3,0 \mathrm{~g} \mathrm{~L}^{-1}\right)$ e, como segundo pré-cultivo, foi utilizado o meio mineral (MM) sem limitação de nitrogênio, descrito por Aragão et al.. ${ }^{19}$

O meio de cultivo final foi composto de uma solução contendo amido hidrolisado e adicionado dos nutrientes que compõem o meio mineral (em g L${ }^{-1}$ ): $\mathrm{KH}_{2} \mathrm{PO}_{4} 1,5, \mathrm{Na}_{2} \mathrm{HPO}_{4} 8,65$, citrato de amônio e ferro III $0,06, \mathrm{CaCl}_{2} .2 \mathrm{H}_{2} \mathrm{O} 0,01, \mathrm{MgSO}_{4} .7 \mathrm{H}_{2} \mathrm{O} 0,5,\left(\mathrm{NH}_{4}\right)_{2} \mathrm{SO}_{4}$ 2,3, ácido nitrilotriacético $0,19 \mathrm{~g} \mathrm{~L}^{-1}$ e elementos traço $1 \mathrm{~mL} \mathrm{~L}^{-1}$. A solução de elementos traço apresentou em sua composição (em $\left.\mathrm{g} \mathrm{L}^{-1}\right) \mathrm{H}_{3} \mathrm{BO}_{3} 0,3, \mathrm{CoCl}_{2} \cdot 6 \mathrm{H}_{2} \mathrm{O} 0,2, \mathrm{ZnSO}_{4} .7 \mathrm{H}_{2} \mathrm{O} 0,1, \mathrm{MnCl}_{2} \cdot 4 \mathrm{H}_{2} \mathrm{O}$ $0,03, \mathrm{Na}_{2} \mathrm{MoO}_{4} \cdot 2 \mathrm{H}_{2} \mathrm{O} 0,03, \mathrm{NiCl}_{2} \cdot 6 \mathrm{H}_{2} \mathrm{O} 0,02 \mathrm{e} \mathrm{CuSO}_{4} \cdot 5 \mathrm{H}_{2} \mathrm{O} 0,01$. A solução simulando resíduo da indústria amilácea foi preparada a partir de arroz branco polido, adquirido no comércio local. $\mathrm{O}$ arroz foi quebrado em liquidificador (doméstico) e depois cozido em água destilada com agitação para obtenção de uma solução concentrada em amido. A hidrólise ácida foi realizada com adição de $\mathrm{HCl}$ 2,7 mol L-1 à solução concentrada de amido, na proporção de 1:10 (ácido:solução com amido). A solução de amido para hidrólise foi aquecida a $110^{\circ} \mathrm{C}$ por 2,5 h, sob pressão de 1,1 atm em autoclave. Após, resfriou-se à temperatura ambiente e neutralizou-se a solução com adição de $\mathrm{NaOH} 2,5 \mathrm{~mol} \mathrm{~L}^{-1}$. O meio foi decantado e centrifugado a $959 x \mathrm{~g}$ por 5 min para a remoção de resíduos sólidos. A solução de amido de arroz hidrolisado foi autoclavada e adicionada ao meio de cultivo para se obter uma concentração inicial de açúcares redutores de $30 \mathrm{~g} \mathrm{~L}^{-1}$.

\section{Condições de cultivo}

Os cultivos foram conduzidos em frascos Erlenmeyer aletados de $1000 \mathrm{~mL}$, contendo $300 \mathrm{~mL}$ do meio de cultivo e incubados em agitador rotatório (shaker) a $150 \mathrm{rpm}$. As temperaturas de cultivo testadas foram de 30,35 e $40{ }^{\circ} \mathrm{C}$, durante o tempo necessário para cada experimento. O meio de cultivo foi inoculado com $10 \%(\mathrm{v} / \mathrm{v})$ do segundo pré-cultivo (MM). $\mathrm{O}$ pH foi mantido em 7,0 pela adição manual de $\mathrm{NaOH} 2,5 \mathrm{~mol} \mathrm{~L}^{-1}$ ou $\mathrm{HCl} 2,7 \mathrm{~mol} \mathrm{~L}^{-1}$.

\section{Adição de suplemento}

A adição de suplemento foi realizada, durante o cultivo, na forma de um pulso no momento da limitação em nitrogênio. O suplemento adicionado foi o óleo de soja esterilizado, contendo $25 \%$ (v/v) de ácido oleico, a fim de se obter uma concentração equivalente a $0,3 \mathrm{~g} \mathrm{~L}^{-1} \mathrm{de}$ ácido oleico no meio.

\section{Técnicas analíticas}

\section{Determinação da concentração celular}

A densidade celular foi determinada por turbidimetria a $600 \mathrm{~nm}$ e a concentração celular por meio de análise gravimétrica. Na análise gravimétrica, amostras de $2 \mathrm{~mL}$ do meio de cultivo foram centrifugadas a $10956 x \mathrm{~g}$ por 3 min e os precipitados foram lavados duas vezes com água destilada e colocados em estufa a $80^{\circ} \mathrm{C}$ até peso constante.

Determinação de açúcares redutores totais (ART)

A determinação dos açúcares redutores totais foi realizada pelo método do ácido 3,5-dinitrossalicílico (DNS). ${ }^{20}$

\section{Nitrogênio residual}

A concentração de nitrogênio residual, na forma de íons amônio, foi determinada pelo método enzimático-colorimérico (kit Ureia-ES - Gold Analisa).

\section{Determinação de $P(3 H B)$}

$\mathrm{O} \mathrm{P}(3 \mathrm{HB})$ foi determinado por cromatografia gasosa, conforme o método de metanólise baseado em Braunegg et al.,$^{21}$ com as modificações propostas por Brandl et al.. ${ }^{22}$ Poli(3-hidroxibutirato) foi usado como padrão externo. Os metil ésteres resultantes da reação de metanólise foram quantificados pela injeção de $1 \mu \mathrm{L}$ de amostra em cromatógrafo a gás (CG-90) equipado com uma coluna de sílica fundida (Supercowax-10 - 0,53 mm x $30 \mathrm{~m}$ ) e detector com ionização de chama. As temperaturas de injeção, detecção e coluna foram 190, 230 e $90{ }^{\circ} \mathrm{C}$, respectivamente.

\section{Tratamento dos dados}

\section{Produtividade média de $P(3 H B)$}

A produtividade média (Equação 1) $\left(\mathrm{P}_{\mathrm{r}}\right)$, expressa em $\mathrm{g} \mathrm{L}^{-1} \mathrm{~h}^{-1}$ foi calculada pela diferença entre a concentração de $\mathrm{P}(3 \mathrm{HB}) \mathrm{em}$ um instante de tempo $\mathrm{t}(\mathrm{P}(3 \mathrm{HB}) \mathrm{t})$ e a concentração inicial de $\mathrm{P}(3 \mathrm{HB})$ $\left(\mathrm{P}(3 \mathrm{HB}) \mathrm{t}_{0}\right)$, dividida pelo intervalo de tempo correspondente, onde $\mathrm{t}_{0}$ é o tempo inicial.

$P_{r}=\frac{P(3 H B) t-P(3 H B) t_{0}}{t-t_{0}}$

\section{Modelagem do crescimento microbiano}

O modelo de Gompertz (Equação 2) ${ }^{23}$ foi ajustado aos dados experimentais da absorbância versus o tempo, a diferentes temperaturas $\left(30,35\right.$ e $\left.40^{\circ} \mathrm{C}\right)$, por meio do software Matlab 7.4.

$\operatorname{Ln}\left(a b s / a b s_{0}\right)=A \cdot \exp \{-\exp [-B(t-M)]\}$

Nesta equação: $\operatorname{Ln}\left(a b s / a b s_{0}\right.$ ) é o logaritmo neperiano do aumento da densidade celular no tempo t, sendo que abs é a densidade celular no tempo zero; $t$ é o tempo (h); abs é a densidade celular no tempo $t$; 
$B$ é a velocidade relativa máxima de crescimento no tempo $\mathrm{M}\left(\mathrm{h}^{-1}\right) ; \mathrm{M}$ o tempo necessário para atingir a velocidade máxima de crescimento

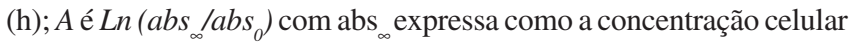
máxima atingida no tempo $\mathrm{t}=\infty$.

Por meio do ajuste realizado pelo modelo de Gompertz, determinaram-se os parâmetros A, B e M, possibilitando o cálculo dos parâmetros de interesse: velocidade específica máxima de crescimento $\left(\mu_{\max }\left(\mathrm{h}^{-1}\right)\right)$ e duração da fase lag $(\lambda(\mathrm{h}))$. Esses parâmetros foram determinados pela aplicação das Equações 3 e 4, respectivamente. O parâmetro aumento logarítmico da população (A) é determinado diretamente pelo ajuste da Equação 1 aos dados experimentais.

$\mu_{\max }=\frac{B \cdot A}{e}$

onde $\mathrm{e}=2,7182$

$\lambda=M-\left(\frac{1}{B}\right)$

\section{RESULTADOS E DISCUSSÃO}

\section{Cultivos a diferentes temperaturas}

O modelo de Gompertz foi ajustado aos dados experimentais de Ln(absorbância/absorbância ) versus o tempo no cultivo de Cupriavidus necator em amido de arroz hidrolisado, às temperaturas de 30 , 35 e $40{ }^{\circ} \mathrm{C}$ sem e com suplementação de óleo de soja. A Figura 1a e $\mathrm{b}$ apresenta os resultados obtidos para estes ajustes. Observa-se que o modelo de Gompertz se ajusta bem a todas as curvas de crescimento, sem ou com suplementação do óleo de soja. Os cultivos apresentaram o mesmo comportamento até o momento da limitação em nitrogênio $\left(12^{\mathrm{a}} \mathrm{h}\right.$, para os cultivos a 30 e $35^{\circ} \mathrm{C}$ ), pois não havia diferença na composição dos meios até esse momento. Na fase de acúmulo do polímero (a partir da $12^{\mathrm{a}} \mathrm{h}$ de cultivo), observou-se uma diferença pouco expressiva para o Ln(absorbância/absorbância ) versus o tempo entre os cultivos sem e com suplementação do óleo de soja. Comparando-se os cultivos submetidos a diferentes temperaturas $\left(30,35\right.$ e $\left.40{ }^{\circ} \mathrm{C}\right)$, nota-se um crescimento característico do microorganimo C. necator para as temperaturas de 30 e $35^{\circ} \mathrm{C}$. O cultivo à temperatura de $40{ }^{\circ} \mathrm{C}$ apresentou um crescimento diferente dos demais cultivos, ocorrendo início da fase estacionária de crescimento antes da limitação de nitrogênio (aproximadamente 6 h de cultivo) esperada para esse cultivo.

A Tabela 1 apresenta os parâmetros $\mu_{\text {máx }}$ (velocidade específica máxima de crescimento), $\lambda$ (duração da fase lag), A (aumento logarítmico da população) e $\mathrm{R}^{2}$ (coeficiente de correlação) obtidos pelo ajuste ao modelo de Gompertz aos cultivos de $C$. necator utilizando amido de arroz hidrolisado como fonte de carbono nas temperaturas de 30,35 e $40{ }^{\circ} \mathrm{C}$, sem e com suplementação de óleo de soja.

Os resultados apresentados na Tabela 1 mostram que os valores de $\mu_{\max }$ para as temperaturas de 30 e $35^{\circ} \mathrm{C}$, nos cultivos sem e com suplementação em óleo de soja, estão de acordo com os valores encontrados na literatura, em que a faixa de crescimento se encontra em torno de $0,22 \mathrm{~h}^{-1} \cdot{ }^{24}$ Porém, no cultivo a $40{ }^{\circ} \mathrm{C}$, observa-se que a velocidade específica máxima de crescimento foi aproximadamente o dobro dos outros cultivos realizados $\left(0,446 \mathrm{~h}^{-1}\right)$. Os cultivos a 30 e $35^{\circ} \mathrm{C}$, com e sem suplementação de óleo de soja, apresentaram praticamente o mesmo tempo de adaptação da célula ao meio de cultivo $(\lambda)$. $\mathrm{O}$ aumento logarítmico da população (A) foi superior na temperatura de $35{ }^{\circ} \mathrm{C}$ para todos os cultivos. Esses resultados indicam que o uso de amido de arroz hidrolisado, como fonte de carbono no crescimento de $C$. necator, à temperatura de $35^{\circ} \mathrm{C}$ se mostra a alternativa mais
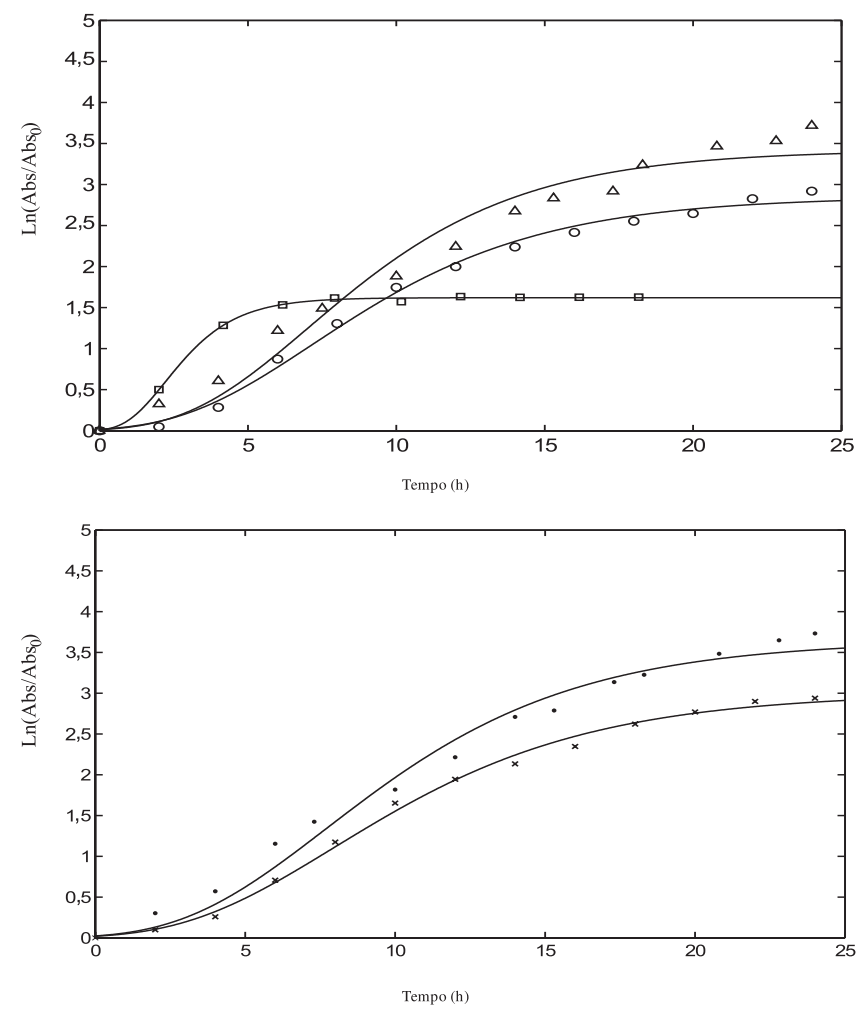

Figura 1. Perfil do crescimento de Cupriavidus necator em meio hidrolisado de arroz expresso em Ln (abs/abs, versus o tempo nas temperaturas de $30{ }^{\circ} \mathrm{C}(\mathrm{O}), 35^{\circ} \mathrm{C}(\Delta)$ e $40{ }^{\circ} \mathrm{C}(\square)$ para os cultivos sem suplementação de óleo de soja $($ a $)$ e, $30^{\circ} \mathrm{C}(\times)$ e $35^{\circ} \mathrm{C}(\bullet) \mathrm{com}$ suplementação do óleo de soja $(b)$. As linhas representam o ajuste do modelo de Gompertz aos dados experimentais

Tabela 1. Tempo de duração da fase lag $(\lambda(h))$, velocidade específica máxima de crescimento $\left(\mu_{\text {máx }}\left(h^{-1}\right)\right)$, aumento logarítmico da população de microorganismos (A) e coeficiente de correlação $\left(\mathrm{R}^{2}\right)$, obtidos a partir do ajuste do modelo de Gompertz aos dados experimentais dos cultivos em amido de arroz hidrolisado como fonte de carbono nas temperaturas de 30,35 e $40^{\circ} \mathrm{C}$, com e sem a adição de suplementação de óleo de soja, para o micro-organismo $C$. necator na produção de poli(3-hidroxibutirato)

\begin{tabular}{llllll}
\hline \multirow{2}{*}{ Parâmetros } & \multicolumn{2}{c}{$\begin{array}{c}\text { Resíduo amiláceo sem } \\
\text { suplementação em óleo de soja }\end{array}$} & \multicolumn{2}{c}{$\begin{array}{c}\text { Resíduo amiláceo } \\
\text { com suplementação } \\
\text { em óleo de soja }\end{array}$} \\
\cline { 2 - 6 } & $30{ }^{\circ} \mathrm{C}$ & $35{ }^{\circ} \mathrm{C}$ & $40{ }^{\circ} \mathrm{C}$ & $30{ }^{\circ} \mathrm{C}$ & $35{ }^{\circ} \mathrm{C}$ \\
\hline$\mu_{\max }\left(\mathrm{h}^{-1}\right)$ & 0,238 & 0,307 & 0,446 & 0,224 & 0,282 \\
$\lambda(\mathrm{h})$ & 2,761 & 2,949 & 0,883 & 3,072 & 2,951 \\
$\mathrm{~A}$ & 2,853 & 3,420 & 1,612 & 3,003 & 3,648 \\
$\mathrm{R}^{2}$ & 0,998 & 0,992 & 0,999 & 0,998 & 0,995 \\
\hline
\end{tabular}

adequada para o crescimento deste micro-organismo, possibilitando altas velocidades específicas de crescimento. Vale destacar que o arroz hidrolisado apresenta concentrações satisfatórias da fonte de carbono metabolizável por $C$. necator, além de possuir em sua composição cerca de 10 a $15 \%$ de proteínas que hidrolisadas servem como fonte de nitrogênio e fósforo para o micro-organimo e, ainda uma pequena porcentagem de lipídeos, fibras e minerais (aproximadamente $1 \%)^{25}$.

Ao se analisar a Figura 1 em conjunto com os dados apresentados na Tabela 1, percebe-se que o crescimento do micro-organismo nos cultivos a 30 e $35^{\circ} \mathrm{C}$ é semelhante e característico de $C$. necator. Inicialmente há um período de fase lag, seguida da fase exponencial e da fase estacionária de crescimento, na qual ocorre a limitação de 
um nutriente essencial ao crescimento celular, indicando o início da fase de produção de $\mathrm{P}(3 \mathrm{HB})$. $\mathrm{O}$ cultivo a $40{ }^{\circ} \mathrm{C}$ apresentou uma menor fase de adaptação das células ao cultivo (duração da fase lag ( $\lambda$ ) ) quando comparado aos cultivos a 30 e $35^{\circ} \mathrm{C}$ e, uma fase exponencial de menor duração, porém com maior velocidade específica de crescimento. No início da fase estacionária para o cultivo a $40{ }^{\circ} \mathrm{C}$ (cerca de 6 h de cultivo, Figura 1) observou-se, por meio da análise de nitrogênio, que a concentração de nitrogênio residual era igual a $0,58 \mathrm{~g}_{\mathrm{N}} \mathrm{L}^{-1}$ neste momento, ressaltando que concentrações em torno de $0,3 \mathrm{~g}_{\mathrm{N}} \mathrm{L}^{-1}$ são consideradas limitantes para $C$. necator. De acordo com estes resultados, não se observou a limitação em nitrogênio para o cultivo a $40^{\circ} \mathrm{C}$. Esses resultados permitem ainda analisar com maior atenção a justificativa de Schmidell, ${ }^{18} \mathrm{em}$ que temperaturas muito altas podem ocasionar uma menor solubilidade do $\mathrm{O}_{2}$ no meio de cultivo, portanto uma limitação da fonte de oxigênio.

\section{Suplementação com óleo de soja em cultivo utilizando resíduo amiláceo como fonte de carbono}

A suplementação com óleo de soja foi realizada no momento da limitação de nitrogênio, com o objetivo de aumentar o conteúdo de $\mathrm{P}(3 \mathrm{HB})$ na célula. Os resultados estão apresentados nas Figuras 2a, b e c e indicam a evolução do conteúdo de $\mathrm{P}(3 \mathrm{HB})$ nos cultivos com e sem suplementação de óleo de soja nas temperaturas de 30, 35 e $40{ }^{\circ} \mathrm{C}$, respectivamente.

A Figura 2a apresenta concentrações de 2,3 e $3,1 \mathrm{~g} \mathrm{~L}^{-1}$ de biopolímero obtidas no final dos cultivos a $30{ }^{\circ} \mathrm{C}$ sem suplementação e com suplementação de óleo de soja, respectivamente. $\mathrm{O}$ mesmo comportamento de aumento da concentração de biopolímero foi observado na Figura $2 \mathrm{~b}$ para o cultivo a $35^{\circ} \mathrm{C}$, em que a concentração de $\mathrm{P}(3 \mathrm{HB})$ para o cultivo sem suplementação foi de $4,3 \mathrm{~g} \mathrm{~L}^{-1} \mathrm{e}$ com suplementação foi de $5,0 \mathrm{~g} \mathrm{~L}^{-1}$. Estes resultados indicam que o experimento a $35^{\circ} \mathrm{C}$ com suplementação de óleo de soja apresentou um aumento de $38 \%$ no conteúdo final de $\mathrm{P}(3 \mathrm{HB})$ na célula (para o mesmo tempo de cultivo) em relação ao cultivo em $30{ }^{\circ} \mathrm{C}$ suplementado com óleo. Portanto, pode-se afirmar que a suplementação com óleo de soja no início da fase de produção potencializa a produção de $\mathrm{P}(3 \mathrm{HB})$ na célula, agindo diretamente na via de síntese deste polímero. Uma possível explicação para este processo metabólico foi dada por Fukui e Dói, ${ }^{26}$ que consideraram que, no momento em que triglicerídeos, presentes nos óleos vegetais, são adicionados ao meio de cultivo ocorre a excreção de enzimas lipases de $C$. necator no meio para hidrolisar estes compostos que, em seguida, são metabolizados intracelularmente por meio da $\beta$-oxidação de ácidos graxos. Uma vez incorporados pela célula, esses ácidos graxos são convertidos ao correspondente acil-CoA tioéster e oxidados pela $\beta$-oxidação dos ácidos graxos via trans-2-enoil-CoA e (S)-3-hidroxiacil-CoA a 3 -cetoacil-CoA, a qual é clivada pela $\beta$-cetotiolase a acetil-CoA e acil-CoA. Em seguida, mais ciclos ocorrem até que a acil-CoA seja completamente convertida a acetil-CoA. Este é o caso para ácidos graxos que apresentam um número de átomos de carbono ímpar em sua cadeia principal e, ainda, ácidos graxos saturados e alifáticos não substituídos. Se o ácido graxo contém grupos funcionais com cadeia lateral acil com carbonos não saturados para ligações carbônicas, a via degradativa pode ser diferente e podem-se requerer enzimas adicionais. Sob condições fisiológicas que permitam a síntese e acúmulo de PHAs (limitação em um nutriente essencial não fonte de carbono), o ácido graxo não se degrada completamente a acetil-CoA e, intermediários da $\beta$-oxidação são parcialmente ou completamente removidos e convertidos nestes poliésteres. No entanto, nenhum dos intermediários é aceito como substrato pela PHA sintase. Por este motivo, é necessária uma ligação metabólica que converta um dos intermediários na $(R)$-3-hidroxiacil-CoA.
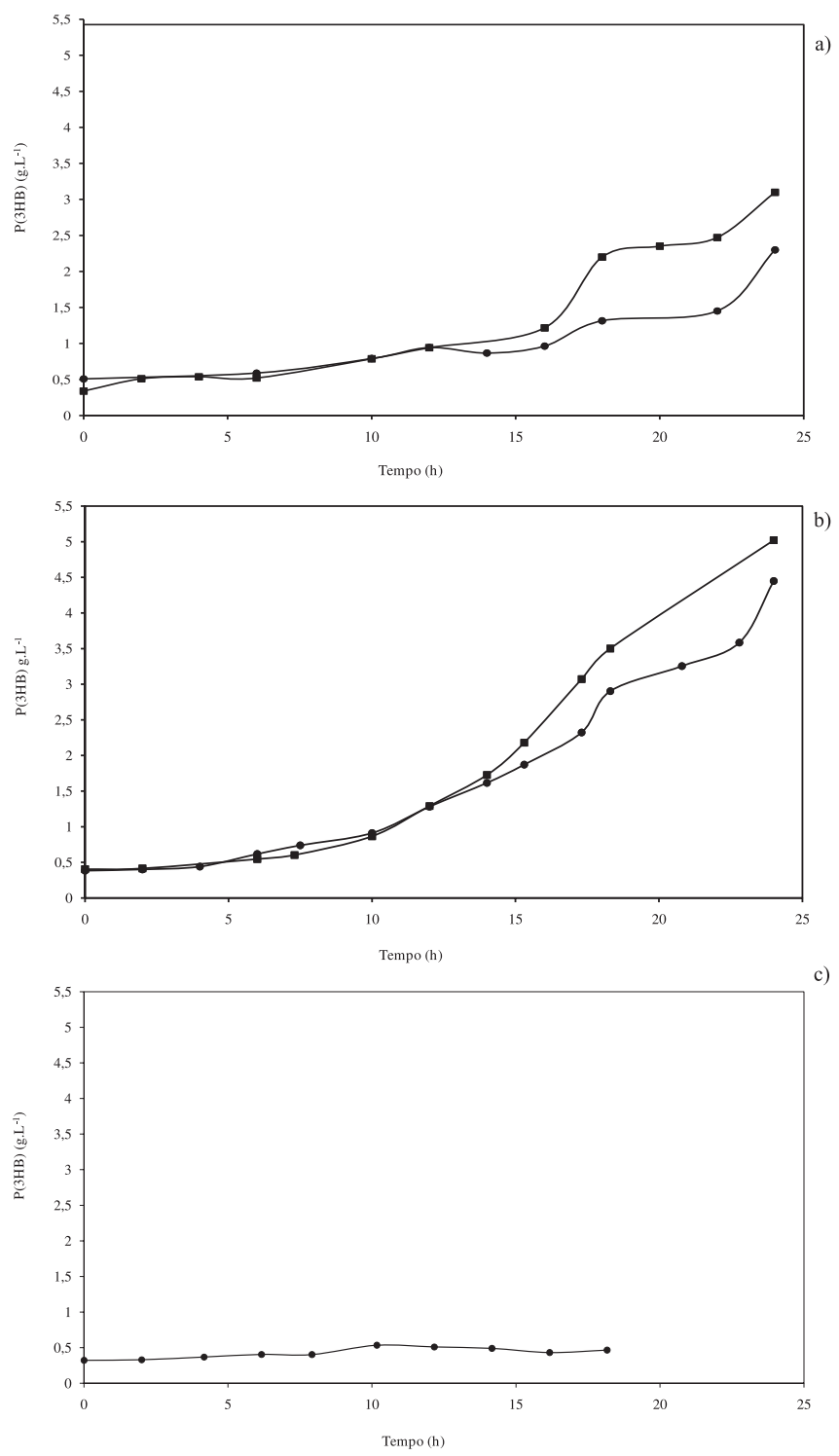

Figura 2. Evolução da produção de $P(3 H B)$ para os cultivos com suplementação de óleo $(-)$ e sem suplementação de óleo $(\bullet)$ nas temperaturas de cultivo de $30{ }^{\circ} \mathrm{C}(a), 35^{\circ} \mathrm{C}(\mathrm{b}) \mathrm{e} 40^{\circ} \mathrm{C}$ (c) (suplementação realizada com $12 \mathrm{~h}$ de cultivo)

De acordo com a Figura 2, observa-se ainda que no cultivo realizado à temperatura de $35^{\circ} \mathrm{C}$, além de apresentar os melhores resultados para crescimento (Tabela 1), mostrou-se também que esta temperatura é a mais adequada para a produção de $\mathrm{P}(3 \mathrm{HB})$ por $C$. necator quando se utiliza amido de arroz hidrolisado como substrato principal.

Na Figura 2c, o cultivo a $40^{\circ} \mathrm{C}$ apresentou baixa concentração de polímero em consequência da transferência prejudicada de oxigênio em cultivos executados em shaker e da baixa concentração celular no momento da limitação do crescimento, possivelmente pela limitação em oxigênio. Vale ressaltar que a limitação em oxigênio é caracterizada por apresentar baixa conversão em polímero e menor produtividade do produto, quando comparada com a limitação de nutrientes como nitrogênio ou fósforo para $C$. necator. ${ }^{28}$ A concentração de $\mathrm{P}(3 \mathrm{HB})$ observada para o cultivo sem suplementação em óleo de soja foi de $0,46 \mathrm{~g} \mathrm{~L}^{-1}$. O cultivo a $40{ }^{\circ} \mathrm{C}$ com suplementação em óleo não foi realizado, visto que o pulso em óleo de soja estava planejado para o momento em que houvesse a limitação do nutriente nitrogênio, evento este que não se concretizou para o referido experimento.

Com base na concentração final de $\mathrm{P}(3 \mathrm{HB})$ e na massa seca final de cada cultivo, foi possível obter a porcentagem de biopolímero para 
Tabela 2. Concentração total de biomassa $\left(\mathrm{Xt}\left(\mathrm{g} \mathrm{L}^{-1}\right)\right)$, concentração total de $\mathrm{P}(3 \mathrm{HB})\left(\mathrm{g} \mathrm{L}^{-1}\right)$, porcentagem de $\mathrm{P}(3 \mathrm{HB})$ na biomassa total $(\% \mathrm{P}(3 \mathrm{HB}))$ e produtividade de $\mathrm{P}(3 \mathrm{HB})\left(\operatorname{Pr}\left(\mathrm{g} \mathrm{L}^{-1} \mathrm{~h}^{-1}\right)\right)$ para o tempo total de cultivo (h) de C. necator em meio hidrolisado de amido de arroz nas temperaturas de 30 e $35^{\circ} \mathrm{C}$ com e sem suplementação de óleo de soja no momento da limitação em nitrogênio

\begin{tabular}{lccccc}
\hline Cultivos & $\begin{array}{c}\text { Tempo } \\
(\mathrm{h})^{\mathrm{a}}\end{array}$ & $\begin{array}{c}\mathrm{Xt} \\
\left(\mathrm{g} \mathrm{L}^{-1}\right)\end{array}$ & $\begin{array}{c}\mathrm{P}(3 \mathrm{HB}) \\
\left(\mathrm{g} \mathrm{L}^{-1}\right)\end{array}$ & $\begin{array}{c}\% \mathrm{P} \\
(3 \mathrm{HB})\end{array}$ & $\begin{array}{c}\mathrm{Pr} \\
\left(\mathrm{g} \mathrm{L}^{-1} \mathrm{~h}^{-1}\right)\end{array}$ \\
\hline $\begin{array}{l}30^{\circ} \mathrm{C} \text { sem adição } \\
\text { de óleo de soja }\end{array}$ & 24,0 & 7,69 & 2,30 & 30,0 & 0,09 \\
$\begin{array}{l}30^{\circ} \mathrm{C} \text { com adição } \\
\text { de óleo de soja }\end{array}$ & 24,0 & 7,80 & 3,09 & 39,0 & 0,13 \\
$\begin{array}{l}35^{\circ} \mathrm{C} \text { sem adição } \\
\text { de óleo de soja }\end{array}$ & 24,0 & 12,40 & 4,34 & 35,0 & 0,18 \\
$\begin{array}{l}35^{\circ} \mathrm{C} \text { com adição } \\
\text { de óleo de soja }\end{array}$ & 24,0 & 11,64 & 5,00 & 43,0 & 0,20 \\
\hline
\end{tabular}

${ }^{a}$ tempo total de cultura.

os experimentos a 30 e $35^{\circ} \mathrm{C}$ com e sem suplementação de óleo de soja. Os dados para o cultivo a $40^{\circ} \mathrm{C}$ não foram apresentados devido à baixa produção de polímero. Os resultados estão apresentados na Tabela 2. Para o cultivo a $35^{\circ} \mathrm{C}$, o acúmulo foi de $35,0 \%$ sem suplementação e $43,0 \%$ com suplementação de óleo de soja.

Na Tabela 2, observa-se que a porcentagem de $\mathrm{P}(3 \mathrm{HB})$ obtida para o cultivo a $30^{\circ} \mathrm{C}$ sem e com suplementação em óleo de soja foi de 30,0 e $39,0 \%$, respectivamente. Estes resultados, quando comparados à porcentagem de $33 \%$ de $\mathrm{P}(3 \mathrm{HB})$ obtida por Akiyama et al. ${ }^{29}$ na suplementação de um cultivo de Alcaligenes $s p$. utilizando $3,0 \mathrm{~g} \mathrm{~L}^{-1}$ de óleo de soja na temperatura de $30^{\circ} \mathrm{C}$, mostram-se mais interessantes. As maiores porcentagens de $\mathrm{P}(3 \mathrm{HB})$ obtidas no presente trabalho foram para os cultivos a $35^{\circ} \mathrm{C}$. Os resultados de porcentagem e produtividade de $\mathrm{P}(3 \mathrm{HB})\left(35 \%\right.$ e $0,18 \mathrm{~g} \mathrm{~L}^{-1} \mathrm{~h}^{-1}$, respectivamente) para o cultivo a $35^{\circ} \mathrm{C}$ sem adição de óleo usando amido hidrolisado de arroz como fonte de carbono são comparáveis aos apresentados por Khanna e Srivastava, ${ }^{30}$ em que uma fonte de carbono sintética (frutose) foi utilizada para cultivos em shaker a $30^{\circ} \mathrm{C}$. Neste trabalho os autores obtiveram uma porcentagem de $\mathrm{P}(3 \mathrm{HB})$, produzido em $48 \mathrm{~h}$ de cultivo, de cerca de $43 \%$ e a produtividade de $0,029 \mathrm{~g} \mathrm{~L}^{-1} \mathrm{~h}^{-1}$. Vale ressaltar que a diferença de produtividade observada pode estar associada à temperatura de $35^{\circ} \mathrm{C}$ e à composição do meio de arroz hidrolisado.

A produtividade observada para o cultivo a $30^{\circ} \mathrm{C}$ com suplementação em óleo foi de $0,13 \mathrm{~g} \mathrm{~L}^{-1} \mathrm{~h}^{-1}$. Esses resultados estão de acordo com os obtidos por Grigull et al. ${ }^{31}\left(0,17 \mathrm{~g} \mathrm{~L}^{-1} \mathrm{~h}^{-1}\right)$, quando estes utilizaram $0,3 \mathrm{~g} \mathrm{~L}^{-1}$ de ácido oleico como suplemento nutricional em cultivos a $30^{\circ} \mathrm{C}$. $\mathrm{O}$ aumento da produtividade, para os experimentos a 30 e $35^{\circ} \mathrm{C}$, em função da suplementação em óleo de soja indica a vantagem em se utilizar baixas concentrações de um suplemento de baixo custo e obter alta produtividade em $\mathrm{P}(3 \mathrm{HB})$.

\section{CONCLUSÕES}

$\mathrm{O}$ cultivo realizado à temperatura de crescimento de $35^{\circ} \mathrm{C}$ apresentou vantagens no crescimento e produção de $\mathrm{P}(3 \mathrm{HB})$ por Cupriavidus necator quando comparado ao realizado à temperatura de $30^{\circ} \mathrm{C}$, atualmente utilizada nos processos de produção deste polímero.

A utilização de amido hidrolisado de arroz mostrou-se uma alternativa potencial para a produção de $\mathrm{P}(3 \mathrm{HB})$ e a suplementação com óleo de soja, mesmo em pequenas quantidades, levou a um aumento na produção de $\mathrm{P}(3 \mathrm{HB})$.

\section{REFERÊNCIAS}

1. Lee, S. Y.; Biotechnol. Bioeng. 1996, 49, 1.

2. Madison, L. L.; Huisman, G. W.; Microbiol. Mol. Biol. Rev. 1999, 63, 21.

3. Ramsay, B. A.; Proceedings of The Symposium on Physiology, Kinetics, Production and Use of Biopolymers, Seggau, Austria, 1994.

4. Khanna, S.; Srivastava, A. K.; Process Biochem. (Amsterdam, Neth.) 2005, 40, 607.

5. Kim, B. S.; Enzyme Microb. Technol. 2000, 27, 774.

6. http://www.abam.com.br/revista/revista12/entrevista.php, acessada em Junho 2009.

7. Yu, J.; J. Biotechnol. 2001, 86, 105.

8. Sarikava, E.; Higasa, T.; Adachi, M.; Mikami, B. Process Biochem. (Amsterdam, Neth.) 2000, 35, 711.

9. Rusendi, D.; Sheppard, J. D.; Bioresour. Technol. 1995, 54, 191.

10. Kim, B. S.; Chang, H. N.; Biotechnol. Lett. 1998, 20, 109.

11. Kahar, P.; Tsuge, T.; Taguchi, K.; Doi, Y.; Polym. Degrad. Stab. 2004, 83,79 .

12. Marangoni, C.; Furigo, J. R., A.; Aragão, G. M. F.; Biotechnol. Lett. 2000, 22, 1635

13. Squio, C. R.; Aragão, G. M. F.; Quim. Nova 2004, 27, 615.

14. Doi, Y., Kawaguchi, Y.; Kunioka, M.; FEMS Microbiol. Lett. 1990, 67, 165.

15. Khanna, S.; Srivasta, A. K.; Biochem. Eng. J. 2005, 27, 197.

16. Lee, Y.; Kim, K. M.; Chang, H. N.; Park, Y. H.; FEMS Microbiol. Lett. 1995, 131, 35 .

17. Schmidell, W. Em Biotecnologia Industrial; Schimidell, W.; Lima, U. A.; Aquarone, E.; Borzani, W., eds.; Edgard Blücher: São Paulo, 2001, vol. 2, cap. 6 .

18. Schmidell, W. Em ref. 17, cap. 14.

19. Aragão, G. M. F.; Lindley, N. D.; Uribellarea, J. L.; Pareilleux, A.; Biotechnol. Lett. 1996, $18,937$.

20. Miller, G. L.; Anal. Chem. (Washington, DC, U. S.). 1959, 31, 426.

21. Braunegg, G.; Sonnleitner, B.; Lafferty, R. M.; Eur. J. Appl. Microbiol. Biotechnol. 1978, 6, 29.

22. Brandl, H.; Gross, R. A.; Lenz, R. W.; Fuller, R. C.; Appl. Environ. Microbiol. 1988, 54, 1977.

23. Erkmen, O.; J. Biosci. Bioeng. 2001, 92, 39.

24. Koyama, N.; Doi, Y.; Biotechnol. Lett. 1995, 17, 281.

25. http://www.farsul.org.br/resources/anexos/comissao/Teconologiadoarroz. pdf, acessada em Julho 2009.

26. Fukui, T.; Doi, Y.; Appl. Microbiol. Biotechnol. 1998, 49, 333.

27. Steinbüchel, A.; Lütke- Eversloh, T.; Biochem. Eng. J. 2003, 16, 81.

28. Lee, S. Y., Choi, J. E.; Wong, H. H.; Int. J. Biol. Macromol. 1999, 25, 31 .

29. Akiyama, M.; Taima, Y.; Doi, Y.; Eur. J. Appl. Microbiol. Biotechnol. 1992, 37, 698 .

30. Khanna, S.; Srivasta, A. K.; Process Biochem. 2005, 40, 2173.

31. Grigull, V. H.; Silva, D. D.; Garcia, M. C. F.; Furlan, S. A.; Pezzin, A. P. T.; Schneider, A. L. S.; Aragão, G. F.; Food Technol. Biotechnol. 2008, 46, 223. 